Revue

Revue de l'histoire des religions

de Ihistoire des religions

Histoire de la littérature grecque chrétienne. Introduction. Sous la responsabilité de Enrico NORELLI et Bernard POUDERON

Paris, Éditions du Cerf, 2008, 332 p., 22 cm (« Initiations aux Pères de l'Église »), $32 €$.

Matteo Grosso

\title{
OpenEdition
}

Journals

Édition électronique

URL : http://journals.openedition.org/rhr/7629

DOI : 10.4000/rhr.7629

ISSN : 2105-2573

Éditeur

Armand Colin

Édition imprimée

Date de publication : 1 octobre 2010

Pagination : 397-400

ISBN : 978-2200-92657-7

ISSN : 0035-1423

Référence électronique

Matteo Grosso, « Histoire de la littérature grecque chrétienne. Introduction. Sous la responsabilité de Enrico norelul et Bernard pouderon », Revue de l'histoire des religions [En ligne], 3 | 2010, mis en ligne le 26 janvier 2011, consulté le 22 septembre 2020. URL : http://journals.openedition.org/rhr/7629 ; DOI https://doi.org/10.4000/rhr.7629

Ce document a été généré automatiquement le 22 septembre 2020.

Tous droits réservés 


\section{Histoire de la littérature grecque chrétienne. Introduction. Sous la responsabilité de Enrico NORELLI et Bernard POUDERON}

Paris, Éditions du Cerf, 2008, 332 p., 22 cm («Initiations aux Pères de l'Église »), $32 €$.

Matteo Grosso

\section{RÉFÉRENCE}

Histoire de la littérature grecque chrétienne. Introduction. Sous la responsabilité de Enrico NORELLI et Bernard POUDERON, Paris, Éditions du Cerf, 2008, 332 p., $22 \mathrm{~cm}$ ( «Initiations aux Pères de l'Église »), $32 €$.

1 La publication d'une nouvelle histoire de la littérature grecque chrétienne ancienne est toujours un événement remarquable; il l'est d'autant plus dans ce cas où ce défi ambitieux est marqué par la décision de consacrer un tome entier à une introduction méthodologique, ce qui en fait sans aucun doute un unicum. Des six tomes dirigés par Bernard Pouderon et destinés à relater le développement de l'histoire littéraire chrétienne de langue grecque des origines au concile de Chalcédoine (451 apr. J.-C.), le premier compte huit essais écrits par autant de spécialistes du domaine : «Histoire de la littérature et histoire des institutions chrétiennes. Quelques considérations de méthode au seuil d'une histoire de la littérature grecque chrétienne ancienne » (Enrico Norelli); «De l'Histoire ecclésiastique à l'Histoire de la littérature grecque chrétienne: une tradition millénaire» (Paolo Siniscalco); "La transmission des écrits littéraires chrétiens» (Rémi Gounelle); "Les formes et modèles littéraires»(Gilles Dorival); "L'évolution dogmatique et son expression » (Marie-Anne Vannier); "L'esthétique » (Marie-Ange Calvet-Sebasti) ; «Les éditions des textes patristiques » (Martin Wallraff) ; 
"Instruments de travail pour l'étude de la littérature grecque chrétienne ancienne " (Benoît Gain). D'après ces titres, on comprend que les thèmes abordés sont de nature diverse, mais tous susceptibles d'introduire à chaque auteur ancien et à son œuvre.

Ce premier tome permet une lecture à différents niveaux : bien qu'il puisse être utilisé avec profit par des lecteurs cultivés souhaitant aborder de manière rigoureuse et scientifique ce monde diversifié qu'est la littérature grecque chrétienne, certaines parties (tel que l'essai introductif du co-éditeur de l'ouvrage, E. Norelli) vont également offrir plusieurs sujets de réflexion aux spécialistes du domaine. De plus, les néophytes en la matière vont tirer profit, sur le plan pratique également, des deux sections finales du volume, dédiées aux éditions de textes patristiques et aux outils de travail. Dans la première, $\mathrm{M}$. Wallraff retrace l'histoire des grandes collections d'éditions de textes en langue originale (Migne, Die Griechischen Christlichen Schriftsteller der ersten [drei] Jahrhunderte, Corpus Christianorum, Sources chrétiennes, etc.), et les situe dans leur contexte socio-culturel d'origine; dans la deuxième, B. Gain propose des listes très simples à consulter, avec les principaux outils (dictionnaires, éditions de textes, répertoires bibliographiques, histoires de la littérature, des doctrines, des institutions chrétiennes, etc.) qui doivent constituer le point de départ de toute recherche scientifique dans ce domaine, et il inclut aussi une liste de sigles et des notes sur les bibliothèques spécialisées dans les pays francophones.

3 Les autres contributions sont en général plus théoriques, et examinent des thèmes fondamentaux, nécessaires à la compréhension des phénomènes liés à la production littéraire paléochrétienne de langue grecque; bien souvent toutefois, elles sont également utiles à la compréhension de la production littéraire chrétienne en langue latine ou dans les autres langues de l'Antiquité. À ce propos, nous nous bornerons à indiquer, de façon générale, le sujet traité par chaque auteur. P. Siniscalco établit un lien idéal entre le présent ouvrage et la tradition des "histoires littéraires" chrétiennes et des «histoires ecclésiastiques » (dont les modèles autorisés sont le De viris illustribus de Jérôme et l' Historia Ecclesiastica d'Eusèbe), esquissant le développement de ces genres à travers plus d'un millénaire. Il s'agit d'une contribution riche en informations et de grande envergure, ce qui nous fait ressentir l'absence, dans ce volume, d'une table des auteurs. R. Gounelle introduit, pour sa part, le thème de la transmission des " écrits littéraires chrétiens » et ses modalités (directe, indirecte, en traduction), mais aussi celui de la perte de maintes œuvres qui n'ont pas été conservées au cours des siècles. Dans un long chapitre, G. Dorival définit les formes et les genres littéraires utilisés par les écrivains chrétiens dès les premières générations, et il présente ensuite la différentiation qui s'est produite en fonction des objectifs poursuivis par les auteurs et qui peut être répartie en cinq ensembles principaux : la littérature de controverse, celle liée à l'exégèse biblique, les traités de théologie, les écrits historiographiques et les œuvres poétiques. L'auteur guide son lecteur dans l'exploration des différents genres, suivant le fil rouge de l'utilisation des formes et modèles littéraires précédents (classiques et juifs) et du degré d'innovation pouvant être attribué aux auteurs chrétiens eux-mêmes : un renouvellement qui convient à la nouveauté du message.

4 M.-A. Vannier offre pour sa part des fondements d'histoire de la doctrine et elle pousse à réfléchir à deux questions théologiques primordiales, très controversées au cours des premiers siècles de l'histoire du christianisme: le développement de la christologie, étroitement liée à la sotériologie, et l'évolution de la théologie trinitaire. Sans un 
minimum de connaissances à ce sujet (ce dont le lecteur cultivé est souvent dépourvu), il est impossible de bien comprendre la plupart des œuvres littéraires chrétiennes. L'étude de M.-A. Calvet-Sebasti traite un sujet exclusivement littéraire, à savoir l'élaboration formelle et esthétique des textes; il s'agit là d'un thème abordé par les auteurs chrétiens de façon singulière et souvent paradoxale. On sait bien, en effet, que ces auteurs privilégiaient le contenu au détriment de la forme, mais il est également intéressant de découvrir que les règles classiques, à maintes reprises refusées par ces auteurs qui privilégiaient la simplicité, ont été empruntées, respectées et souvent révisées. Reprenant ce qu'a écrit G. Dorival à propos des genres littéraires, M.-A. CalvetSebasti souligne que la double matrice qui est à l'origine de la culture chrétienne débouche sur un renouvellement - stylistique dans ce cas - des traditions antérieures.

Passons finalement à l'essai d'E. Norelli, qui ouvre le volume en examinant certaines questions fondamentales mais trop souvent éludées dans d'autres entreprises du genre. Fort de son expérience et de son équilibre - n'oublions pas qu'il est l'auteur, avec Claudio Moreschini, d'une célèbre Storia della letteratura cristiana antica greca e latina (Brescia, 1993-1996, traduction française du premier tome : Genève, 2000) -, il examine les principaux problèmes épistémologiques de la discipline, et se fonde tout d'abord sur le fait que, par définition, « la littérature est une communication réalisée par des textes écrits» (p. 10), mais qu'elle est souvent liée, surtout dans les sociétés anciennes, à l'oralité. Dans son essai d'environ soixante pages, l'auteur aborde de nombreuses questions; entre autres, il nous rappelle que la recherche historico-littéraire sur les textes proto-chrétiens devrait s'affranchir des critères théologiques de regroupement (en particulier de la catégorie "Nouveau Testament ", qui n'a aucune justification ni sur le plan historique, ni sur le plan littéraire, p. 21). Pour ce qui est de la sélection de la matière faisant l'objet de cette discipline, il souligne l'importance non seulement de la littérature "de haut niveau", mais aussi d'autres formes de communication, qui peuvent éclairer le sens et favoriser la compréhension des œuvres de génie. Très détaillées sont aussi les remarques faites sur la définition de l'adjectif "chrétienne " référé à la littérature, et l'auteur suggère à ce propos d'inclure dans cette catégorie les "écrits qui adoptent Jésus et son enseignement comme référence» (p. 35). Relativement à la perspective dans laquelle la matière devrait être présentée, Norelli reprend la théorie formulée au début du $\mathrm{xx}^{\mathrm{e}}$ siècle par Hermann Jordan, à savoir le fait qu'une " histoire de la littérature est en premier lieu une histoire des formes » (p. 46, reprenant ses mots exacts); il associe toutefois à cette approche une conscience historique jugée nécessaire, et qui doit mettre l'accent sur la transformation des formes, compte tenu du développement des institutions et des rapports de pouvoir.

6 En conclusion, une remarque, même marginale, s'impose à propos des difficultés engendrées par la différenciation, désormais séculaire, entre études néotestamentaires et études patristiques et ses répercussions sur des ouvrages tels que celui qui fait l'objet de la présente recension. Norelli affirme à juste titre qu'il ne convient pas d'aborder séparément les œuvres intégrées dans le corpus du Nouveau Testament et le reste de la production littéraire chrétienne ancienne; en revanche, les sections de ce volume qui traitent de la transmission, de l'édition des textes et des instruments de travail ne les incluent pas. Ce choix se justifie par le fait que les modalités de transmission des textes canoniques ont été singulières et que, de nos jours, elles ont conduit à l'élaboration d'outils de travail adaptés à leur objet d'étude; nous constatons toutefois que, dans la pratique, certains cas obligent à continuer d'utiliser les catégories traditionnelles. Une conscience critique s'impose donc. La lecture et l'étude de ce volume vont sans aucun 
doute contribuer à affiner cette qualité chez bon nombre de jeunes (et moins jeunes) lecteurs.

\section{AUTEURS}

MATTEO GROSSO

Université de Turin. 